\title{
Incidencia de la operación cesárea según la clasificación de Robson en el Servicio de Ginecología y Obstetricia del Hospital Gral. Dr. Fernando Quiroz Gutiérrez del Instituto de Seguridad Social al Servicio de los Trabajadores del Estado
}

\author{
Incidence of cesarean section according to Robson's classification in the Department of \\ Gynecology and Obstetrics of the General Hospital Dr. Fernando Quiroz Gutiérrez, \\ ISSSTE
}

Aidé I. Manny-Zitle y José M. Tovar-Rodríguez*

Servicio de Obstetricia y Ginecología, Hospital Gral. Dr. Fernando Quiroz Gutiérrez, Instituto de Seguridad Social al Servicio de los Trabajadores del Estado (ISSTE), Ciudad de México, México

\begin{abstract}
Resumen
Introducción: El número de cesáreas en todo el mundo se ha incrementado de forma importante y nuestro país no es la excepción. En algunos lugares se supera el $60 \%$ de cesáreas en relación al parto vaginal. No existe una clasificación adecuada para el análisis de este incremento. La clasificación de los 10 grupos de Robson se basa en cuatro pilares: a) antecedentes obstétricos; b) progreso del parto; c) categoría del embarazo; y d) edad gestacional. Sugerimos utilizar la clasificación de Robson para determinar qué grupo de mujeres embarazadas contribuyen más al total de cesáreas en nuestra institución. Método: Estudio retrospectivo, descriptivo, transversal, observacional, de 2014 al 2016, incluyendo a todas las embarazadas con más de 27 semanas de gestación. Se resolvió el embarazo a 706 mujeres con un porcentaje de cesárea del 65.29\%. El promedio de edad fue de $31 \pm 4.2$ años, y el de la edad gestacional fue de $38.5 \pm 6.7$ semanas. Eran primigestas el $47 \%$. La indicación materna más frecuente de cirugía fue por cesárea iterativa, y la fetal por distocia de presentación. En cuanto a la ubicación del mayor número de pacientes dentro de la clasificación de Robson, fue el grupo 5 con un $21.24 \%$, seguido del grupo 2 con un 13.88\% y del grupo 1 con un 9.6\%. Conclusión: La existencia de cicatriz uterina previa fue el factor determinante en la mayor parte de las cesáreas. Sugerimos incidir sobre la indicación de la primera cesárea y así disminuir cicatrices uterinas.
\end{abstract}

PALABRAS CLAVE: Embarazo. Cesárea. Clasificación de Robson.

\begin{abstract}
Introduction: The increase of cesarean sections worldwide has increased significantly, our country is no exception, in some places it exceeds $60 \%$ of cesarean sections in relation to vaginal delivery, there is no adequate classification for the analysis of this increase. The classification of the 10 groups of Robson is based on four pillars: a) obstetric history; b) progress of labor; c) category of pregnancy; and d) gestational age. We suggest using Robson's classification to determine which group of pregnant women contribute most to the total number of cesareans in our institution. Method: This retrospective, descriptive, cross-sectional, observational study was conducted from 2014 to 2016, all pregnant women with more than 27 weeks' gestation were included. Pregnancy was resolved in 706 women with a caesarean section of $65.29 \%$, mean age was $31 \pm 4.2$ years,
\end{abstract}

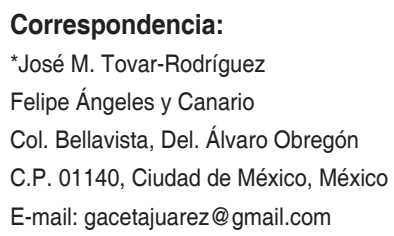

Fecha de recepción: 13-12-2017

Fecha de aceptación: 24-04-2018 DOI: 10.24875/CIRU.M18000044
Cir Cir. 2018;86:261-269

Contents available at PubMed www.cirugiaycirujanos.com 
and gestational age $38.5 \pm 6.7$ weeks. $46.74 \%$ were primiparous. The most frequent maternal indication for surgery was by iterative cesarean section and the fetal one was due to presentation dystocia, as for the location of the patients within the Robson classification was group 5 with $21.24 \%$, group 2 with $13.88 \%$ and the 1 with $9.6 \%$. Conclusion: The previous uterine scarring was the determining factor in most of the cesarean sections, we suggest to influence the indication of the first cesarean section and thus to avoid uterine scars.

KEY WORDS: Pregnancy. Caesarean section. Robson classification.

\section{Introducción}

Los porcentajes de cesáreas han aumentado de forma sustancial, particularmente en los países desarrollados y sobre todo durante la última década. Este continuo aumento ha provocado un debate sobre el porcentaje adecuado de cesáreas, el derecho a elegir o no un parto por cesárea en ausencia de indicación médica, el aumento de los riesgos potenciales para la madre y el feto que podría significar implementar una política de reducción del número de cesáreas, y la preocupación acerca de la sobremedicalización y el abuso de los recursos. La Organización Mundial de la Salud (OMS) declaró en 1985: «No hay justificación para que ninguna región tenga un porcentaje de cesáreas superior al 10-15\%»'. Sin embargo, la monitorización general de los porcentajes de cesáreas es insuficiente para entender la esencia del problema y sus causas. El análisis de información más profunda, como qué grupo de mujeres o de poblaciones obstétricas están siendo sometidas a cesáreas, arrojará más luz acerca de las intervenciones que podrían reducir los porcentajes de cesáreas cuando y donde sea apropiado². La ausencia de metodologías estandarizadas internacionalmente aceptadas ha impedido las comparaciones y la monitorización de los porcentajes de cesáreas de una forma significativa en todo el mundo ${ }^{3}$. En 2001 hubo una propuesta para la vigilancia y la auditoría de la tasa de cesáreas orientada hacia la acción: la clasificación de diez grupos propuesta por Robson, que resulta aplicable internacionalmente y permite comparaciones en el tiempo de una misma unidad asistencial y entre distintas unidades. Esta clasificación proporciona la base para cambiar las prácticas en grupos de mujeres específicos y prospectivamente identificables, obteniéndose un sistema de clasificación útil y clínicamente relevante para mejorar los resultados. Además, la clasificación de diez grupos es robusta debido a que los grupos son mutuamente excluyentes y totalmente incluyentes, por lo que cada mujer puede ser clasificada en un solo grupo y cada una tiene un grupo en el que ser incluida ${ }^{4}$. Esto permite superar un problema que aparece con frecuencia cuando se clasifica la cesárea de acuerdo con la indicación. Esta clasificación está basada en parámetros bien definidos y fáciles de identificar en cada mujer y su embarazo en lugar de la indicación de la cesárea, evitándose así mucha confusión, discrepancia y variabilidad inherente a la indicación de una cesárea. Cuatro pilares forman la base de esta clasificación: a) los antecedentes obstétricos; b) el progreso del parto; c) la categoría del embarazo; y d) la edad gestacional ${ }^{5}$.

\section{Descripción de la clasificación de diez grupos}

- Grupo 1: nulíparas con un feto único en presentación cefálica, de 37 semanas o más de embarazo, que han iniciado el parto de forma espontánea.

- Grupo 2: nulíparas con un feto único en presentación cefálica, de 37 semanas o más de embarazo, que han sido sometidas a inducción o a cesárea antes del inicio del parto.

- Grupo 3: multíparas sin cesárea previa, con un feto único en presentación cefálica, de 37 semanas o más de embarazo, que han iniciado el parto de forma espontánea.

- Grupo 4: multíparas sin cesárea previa, con un feto en presentación cefálica, de 37 semanas 0 más de embarazo, que han sido sometidas a inducción o a cesárea antes del inicio del parto.

- Grupo 5: multíparas con al menos una cesárea previa, con un feto único en presentación cefálica, de 37 semanas o más de embarazo.

- Grupo 6: nulíparas con un feto único en presentación podálica.

- Grupo 7: multíparas con un feto único en presentación podálica, incluyendo aquellas con cesárea previa.

- Grupo 8: todas las mujeres con embarazo múltiple, incluyendo aquellas con cesárea previa.

- Grupo 9: todas las mujeres con feto único en posición transversa u oblicua, incluyendo aquellas con cesárea previa. 
- Grupo 10: todas las mujeres con un feto único en presentación cefálica de menos de 37 semanas de embarazo, incluyendo aquellas con cesárea previa.

\section{Marco teórico}

La operación cesárea surgió alrededor del año 1880 para contribuir a la disminución de la morbimortalidad materna y fetal por desproporción cefalopélvica, cuya mortalidad en ese momento excedía el $80 \%$. A principios de 1900 se implementó en casos de placenta previa o eclampsia y se convirtió en un procedimiento alternativo más seguro que la craneotomía, la sinfisiotomía o la utilización de fórceps altos en trabajos de parto distócicos. A partir de entonces se fueron ampliando las indicaciones e incrementando su uso. Al principio de los años 1970, la proporción de cesárea en los países desarrollados estaba alrededor del $5 \%$, y al final de los años 1990 ya superaba el 50\% en algunas regiones del mundo. Muchos factores contribuyeron a este incremento: mejorías en las técnicas quirúrgicas y anestésicas, reducción de las complicaciones posoperatorias en general, disponibilidad de antibióticos, factores nutricionales y demográficos, percepción de seguridad del procedimiento por parte de las mujeres y del personal de salud, implementación de una práctica médica a la defensiva de los litigios legales, cambios en los sistemas de salud, solicitud de la paciente como un derecho a decidir la vía de nacimiento de su hijo, aplazamiento de la edad para embarazarse, incrementos en el índice de masa corporal materna, aumento de la frecuencia de embarazos múltiples y disminución del parto vaginal después de una primera cesárea ${ }^{7}$.

\section{Sistemas de clasificación para cesáreas}

Las clasificaciones en general se basan en la identificación de diferentes conceptos que pueden tener varios parámetros. Las permutaciones de estos parámetros y su organización sistemática generan grupos o categorías específicas que comparten algunas propiedades, características o cualidades definidas ${ }^{8}$. Para que los datos y la información que se recolectan en los sistemas de salud sean útiles para mejorar los resultados en salud de las personas es necesario organizarlos, y ese es el papel de las clasificaciones: organizar información y datos crudos para transformarlos en conocimiento utilizable.
Cada sistema de clasificación tiene su objetivo y con base en él se estructura. El objetivo más frecuente de las clasificaciones en medicina es mejorar la calidad de la atención y los resultados. Los sistemas de clasificación son exitosos solo si los datos o la información que se recolectan y analizan cumplen con los principios de recolección de la información?.

La información debe ser útil, cuidadosamente definida y recolectada, oportuna y disponible. Las categorías generadas dentro de las clasificaciones también deben cumplir ciertos requisitos: tienen que ser mutuamente excluyentes y totalmente inclusivas. La clasificación debe ser simple de entender e implementar, reproducible y que se pueda hacer de manera prospectiva para generar intervenciones.

Existen varias clasificaciones de la cesárea, algunas basadas en la indicación y en la urgencia de la cesárea, en las condiciones clínicas y obstétricas de las mujeres, y otras en criterios variados ${ }^{10}$. En una revisión del tema se encontraron 27 clasificaciones que se sometieron a un proceso de evaluación que tuvo en cuenta la facilidad de aplicación, la claridad, la reproducibilidad, la posibilidad de implementación, el cumplimiento de tener categorías mutuamente excluyentes y totalmente inclusivas, y la posibilidad de aplicarlas de manera prospectiva. Solo una clasificación obtuvo el máximo puntaje posible, la clasificación de los 10 grupos de Robson, la cual pertenece al grupo de clasificaciones con base en las características de las mujeres $^{11,12}$. Dentro de las clasificaciones basadas en la clasificación de la urgencia, la que obtuvo el máximo puntaje (9 de 14 puntos posibles) fue la de van Dillen, y dentro de las clasificaciones basadas en la indicación de la cesárea, dos tuvieron el máximo puntaje $(9 / 14)$, la de Althabe y la de Anderson.

\section{Clasificación de Robson}

El doctor Michel Robson cita como primera referencia de su clasificación un artículo que publicó en el año 2001'13; sin embargo, en un estudio previo el autor utilizó las mismas categorías de su sistema de clasificación para describir la población y presentar los resultados de un proceso de auditoría en el año 1996, aunque en él no le da identidad al modelo de clasificación. A partir de la publicación del año 2001, la clasificación se ha utilizado ampliamente en el mundo y se ha aplicado tanto en el ámbito institucional como en el regional y con diferentes objetivos ${ }^{14}$. 
La clasificación de Robson se basa en cuatro conceptos obstétricos: los antecedentes obstétricos, el progreso del trabajo de parto, la categoría del embarazo y la edad gestacional; con ellos se clasifican las mujeres en uno de 10 grupos. Las mujeres se agrupan, de acuerdo con la categoría del embarazo, en único con la presencia de un feto en la cavidad uterina y múltiple cuando hay más de uno; según la presentación fetal al momento del parto, en cefálico o de nalga, oblicuo o transverso; según los antecedentes obstétricos, en nulípara si la paciente no tiene parto previo y multípara si tiene más de un parto previo. Además, se consideran el antecedente de cirugía abdominal (con o sin cicatriz uterina), el proceso de trabajo de parto (inducido con medicación o espontáneo) y la edad gestacional al momento del parto (de término si es $\geq 37$ semanas y pretérmino si es $<37$ semanas).

Debido a la reconocida falta de confiabilidad de la fecha de la última menstruación y el margen de error de las ecografías para estimarla con el progreso de la gestación, si se encuentra un recién nacido con una edad gestacional estimada inferior a 37 semanas y con un peso superior al percentil 95 para la semana $36(3093 \mathrm{~g})$ se reclasificará en el grupo de recién nacido de 37 semanas o más ${ }^{15}$.

La clasificación tradicional de las tasas de cesárea a partir de las indicaciones de esta no es recomendable debido a la dificultad de hacerlo de manera prospectiva sin sesgos de registro. La intención de la clasificación de Robson es identificar grupos con mayor o menor probabilidad de terminar en cesárea para tomar medidas antes de que ocurra el evento ${ }^{16}$.

Las características de este sistema de clasificación permiten la comparación en el tiempo en una sola unidad y entre diferentes unidades, proporcionando razones para cambiar prácticas en grupos de mujeres específicos de manera prospectiva. Permite identificar cuáles son los grupos que incrementan las tasas de cesárea, reconociendo de antemano que unos son susceptibles de intervención y otros no, y permite reevaluar la indicación y la pertinencia de la cesárea, e identificar factores de riesgo modificables en estos grupos de pacientes, con lo cual pueden implementarse estrategias de intervención.

Robson reconoce que su clasificación es un paso inicial y que según los resultados deben implementarse adiciones ${ }^{17}$. En la revisión sistemática de la clasificación de Robson del año 2014 se consideraron las recomendaciones de los usuarios de esta clasificación, quienes propusieron realizar subclasificaciones de los grupos o nuevas clasificaciones, inclusión de otras variables y características maternas dentro de cada grupo, además de la necesidad de entrenamiento del personal de salud para una adecuada obtención de los datos para la clasificación de las pacientes ${ }^{18}$.

\section{Usos de la clasificación de Robson}

El objetivo para el cual se ha utilizado más frecuentemente la clasificación es para caracterizar la población atendida e identificar los grupos que más aportan a la proporción de la cesárea o al incremento de ella. Esto se ha hecho para una sola institución, para varias instituciones, para una ciudad, para una región de un país, para varias regiones e incluso para varios países ${ }^{18}$. En algunos estudios, adicional a esta descripción, la utilizan para evaluar tendencias en el tiempo, tanto en el ámbito institucional como en el poblacional. Algunos autores avanzan más allá de la descripción y utilizan la clasificación para realizar comparaciones entre instituciones, identificar diferencias entre ellas y buscar las posibles causas de la variabilidad en la proporción de cesáreas, e incluso evalúan el efecto de disminuir esa variabilidad tanto en una región como entre diferentes regiones o en grupos poblacionales ${ }^{19}$. Algunos estudios evalúan diferencias dentro de los grupos y exploran factores adicionales a los contemplados en la clasificación como explicación a las cifras de cesárea, a sus aumentos o a su correlación con los indicadores de morbimortalidad $^{20}$. También se ha utilizado como herramienta para estandarizar comparaciones entre instituciones y para cuantificar el efecto de intervenciones para disminuir la frecuencia de la cesárea. La clasificación se ha empleado para cumplir objetivos más allá de la cesárea, como es el estudio de la morbilidad obstétrica grave, o para estudiar subgrupos específicos de la clasificación. En México no existe al menos una experiencia publicada con la clasificación, y por lo tanto no se ha estimado la proporción de cesárea esperada para una institución acorde con el tipo de población atendida utilizando la clasificación para realizar una estandarización de tasas.

La clasificación de diez grupos ha sido instaurada en diferentes unidades obstétricas en el Reino Unido, Canadá, África, Suecia, los EE.UU. y Chile, entre otros países.

En mayo de 2015 la OMS publicó un análisis secundario de dos encuestas multinacionales, la Encuesta Mundial de Salud Materna y Perinatal de la OMS (WHOGS; 2004-08) y la Encuesta Multinacional de la 
OMS de Salud Materna y Neonatal (WHOMCS; 2010-11), en las que se analizaron datos de 21 países que fueron incluidos para evaluar las tendencias de la operación de cesárea. Se utilizaron los datos de estas encuestas para establecer el porcentaje de cambio anual promedio en las tasas de cesárea por país, y se encontró que el uso de los criterios de Robson permite comparaciones estandarizadas de los datos entre países y momentos de tiempo, e identifica las subpoblaciones que impulsan los cambios en las tasas de cesárea, por lo que recomiendan ampliamente su uso. Haber tenido una cesárea anteriormente es un determinante cada vez más importante de las tasas generales de cesárea en los países con un índice de desarrollo humano moderado o bajo. Las estrategias para reducir la frecuencia del procedimiento deben incluir la disminución de la primera cesárea y la mejor selección de casos para la inducción del parto y la cesárea en embarazos pretérmino.

En México, actualmente no se ha unificado la utilización de un sistema de clasificación de cesáreas, pero algunas instituciones de salud utilizan la clasificación de cesárea de urgencia, dependiendo del grado de compromiso materno o fetal, estableciendo parámetros de seguridad según el tiempo entre el diagnóstico y el inicio del procedimiento quirúrgico. Se cuenta con estudios en los que se encontró que la aplicación del modelo de Robson para la clasificación de cesárea es fácil y útil para identificar las mujeres, los procedimientos y las prácticas posiblemente susceptibles y prioritarias de intervenir con el fin de tener una proporción racional de cesáreas adaptada a las condiciones propias de la institución y de las mujeres atendidas en ella.

\section{Planteamiento del problema}

Cuando la gestante y sus familiares acuden a urgencias de los establecimientos de salud, llevan consigo una duda respecto al nacimiento de su hijo: ¿el nacimiento será por parto o cesárea?

Por definición, el parto es un proceso fisiológico que la mayoría de las veces la gestante experimenta sin inconvenientes, y si se presentan complicaciones, estas usualmente se dan de forma rápida e inesperada y deben ser previstas por el profesional. La cesárea, al ser una intervención médica quirúrgica, implica un riesgo inherente a la intervención; en este caso pueden ser complicaciones derivadas del acto quirúrgico o efectos secundarios del acto anestésico, pero cuando la intervención está indicada estos riesgos se encuentran por debajo de los beneficios de realizar la cesárea, no siendo así cuando la cesárea se realiza sin una indicación médica clara.

La intervención por cesárea, cuando está justificada, es eficaz para prevenir la morbimortalidad materna y perinatal. Sin embargo, la OMS informa de que una tasa ideal de cesárea no debe exceder el 10-15\%, y que no están demostrados los beneficios de la cesárea en las pacientes en las que este procedimiento es innecesario y conlleva riesgos inmediatos y posteriores, afectando la salud del binomio madre-niño y la futura salud reproductiva de la mujer.

Es evidente que la tasa de cesáreas en el mundo se ha incrementado. La OMS indica que Brasil es el país que está en la cima, con un $56 \%$ de partos bajo esta modalidad, y le siguen Egipto con un $51.8 \%$, Turquía con un $47.5 \%$, Italia con un $38.1 \%$; varios países de América Latina se encuentran en estos porcentajes de cesáreas.

En México existe el interés de estudiar y poner al descubierto este problema, que existe e interviene de forma alarmante en la salud de la mujer. Esta práctica casi habitual de la cesárea ha sobrepasado tal medida que se ha decidido estrechar los controles sobre este tipo de cirugías para hacer disminuir su número. En nuestro país es poca la investigación sobre la cesárea indicada por el profesional de la salud que es innecesaria clínicamente, y con ello no se conoce en sí el porqué de la decisión medica sobre la preferencia de la gestante, incluyendo el decidir evitar o no un parto natural, elevando el riesgo y minimizando el de la cesárea, y afectando la salud reproductiva de la mujer.

A ello se suma el vacío que implica no tener un sistema estandarizado que clasifique de forma internacional las tasas de cesáreas, con las tendencias y las causas subyacentes, y por ende su comprensión, monitoreo y comparación. En el año 2001 se propuso el modelo de clasificación de Robson, conocido también como clasificación de 10 grupos, y luego de varios estudios que lo avalan fue incorporado por la OMS como una herramienta para controlar y evaluar las tasas de cesárea, debido a su simplicidad y validez de su propósito, y lo fácil de su aplicación e interpretación. Así, la información recopilada de forma estandarizada, uniforme y reproducible es elemental para el establecimiento de una mejoría en la salud, con el objetivo de optimar el uso de la intervención por cesárea, evaluar y mejorar la calidad de la atención.

La cesárea es el procedimiento quirúrgico más frecuente en todo el mundo, y aunque se es consciente de que salva vidas, muy a menudo se practica $\sin$ 
que haya una indicación sustentable, suceso que pone a la gestante y su neonato en riesgo de sufrir algún desenlace fatal en su salud. Es por eso que resulta fundamental recopilar información sobre el uso de la cesárea en los distintos niveles de atención, sobre todo en segundo nivel, y así poder evaluar, mejorar y optimizar la calidad de la atención para identificar los grupos que más aportan a la cifra global de cesárea, e implementar prácticas en esos grupos de mujeres específicos y prospectivamente identificables, obteniendo un sistema de clasificación útil y clínicamente relevante para mejorar los resultados.

El objetivo del presente trabajo es determinar la incidencia de cesárea utilizando la clasificación de cesáreas de Robson, durante los años 2014 a 2016 en el Hospital General Dr. Fernando Quiroz Gutiérrez del Instituto de Seguridad Social al Servicio de los Trabajadores del Estado.

\section{Método}

El estudio se realizó en el Hospital General Dr. Fernando Quiroz Gutiérrez, de segundo nivel de atención. Es una institución de mediana y alta complejidad encargada de la atención de gestantes y neonatos de bajo, mediano y alto riesgo, que atiende pacientes derechohabientes al servicio del Estado, de la zona poniente de la Ciudad de México. El diseño utilizado fue retrospectivo, transversal y descriptivo. Para el análisis de los resultados se utilizaron medidas de tendencia central y de dispersión; los resultados se presentan en tablas y gráficas.

Se incluyeron todas las mujeres que acudieron a resolución obstétrica por vía vaginal o por cesárea atendidas en el servicio de ginecología y obstetricia entre enero de 2014 y enero de 2016. Se incluyeron todas las pacientes con embarazo de más de 27 semanas. Se extrajeron los datos retrospectivamente de las historias clínicas del hospital en el período estudiado. Se utilizó la clasificación de diez grupos propuesta por Robson. Se obtuvo la información necesaria de cada mujer embarazada para esta clasificación: a) embarazo con feto único/múltiple; b) paridad; c) inicio del parto; d) edad gestacional; e) cesárea o cicatriz uterina previa; f) presentación y situación fetal; y también se tuvo en cuenta la edad materna. Se consideró el inicio del trabajo de parto como espontáneo cuando no hubo intervención médica para desencadenar el inicio de las contracciones uterinas; inducido, cuando se dio una intervención farmacológica para hacerlo; y sin trabajo de parto, cuando se programó la cesárea antes de que ocurriera uno de esos dos eventos. El antecedente de cicatriz uterina incluyó cesárea o miomectomía previa. La edad gestacional se definió por la ecografía más temprana o por la amenorrea anotada al momento del parto. De la historia clínica se tomó el diagnóstico para la indicación de la terminación de la gestación o la realización de la cesárea. La tasa de cesáreas se calculó como la proporción de pacientes que tuvieron parto por cesárea del total de los partos atendidos en el lapso de tiempo.

Las pacientes se clasificaron de acuerdo con los diez grupos clínicos, mutuamente excluyentes, descritos por Robson. Se calculó la relación entre las pacientes pertenecientes a cada grupo de Robson con respecto al total de la población atendida ( $\left[n .^{\circ}\right.$ de pacientes atendidas pertenecientes a cada grupo/n. ${ }^{\circ}$ total de pacientes atendidas, tanto por vía vaginal como por cesárea] $\times 100$ ), lo cual representa el tamaño relativo de cada uno de los grupos.

Se calculó la proporción específica de cesáreas realizadas dentro de cada grupo ([n. ${ }^{\circ}$ de cesáreas realizadas a mujeres pertenecientes a cada grupo $/ n{ }^{\circ}$ de nacimientos, vaginales y por cesárea, pertenecientes a ese mismo grupo] $\times 100$ ).

Para cuantificar la contribución hecha por cada grupo al porcentaje general de partos se dividió el número de cesáreas en el grupo por el total de partos atendidos en el periodo ([n. ${ }^{\circ}$ de total cesáreas realizadas $/{ }^{\circ}{ }^{\circ}$ total de nacimientos, vaginales y por cesárea] $\times 100$ ).

\section{Aspectos éticos}

El presente estudio es retrospectivo a partir de historias clínicas y registros médicos, y se preservaron la confidencialidad, el anonimato y los derechos de las mujeres participantes, considerándose una investigación sin riesgo ya que el sujeto de investigación no sufre daño alguno como consecuencia inmediata o tardía del estudio, según el reglamento de la Ley General de Salud en Materia de Investigación, Título segundo, de los Aspectos Éticos de la Investigación en Seres Humanos, Capítulo I, artículo 17-I.

\section{Resultados}

En el periodo de enero de 2014 a enero de 2016 se atendieron 706 nacimientos, de los cuales el $65.29 \%$ fueron por cesárea.

El promedio de edad de las mujeres fue de $31 \pm 4$ años, y el de la edad gestacional fue de 38.5 semanas. 
Tabla 1. Distribución de las cesáreas según la clasificación de Robson

\begin{tabular}{lccc}
\hline Grupos & Tamaño del grupo & Proporción específica de cesáreas & $\begin{array}{c}\text { Contribución de las cesáreas en el grupo } \\
\text { al total de la atención (\%) }\end{array}$ \\
\hline Grupo 1 & $130(18.41 \%)$ & $68 / 130(52.30 \%)$ & 9.6 \\
Grupo 2 & $128(18.13 \%)$ & $98 / 128(76.56 \%)$ & 13.88 \\
Grupo 3 & $145(20.53 \%)$ & $29 / 145(20 \%)$ & 4.1 \\
Grupo 4 & $47(6.65 \%)$ & $30 / 47(63.82 \%)$ & 4.2 \\
Grupo 5 & $165(23.37 \%)$ & $150 / 165(90.90 \%)$ & 21.24 \\
Grupo 6 & $9(1.27 \%)$ & $9 / 9(100 \%)$ & 1.2 \\
Grupo 7 & $20(2.83 \%)$ & $20 / 20(100 \%)$ & 2.83 \\
Grupo 8 & $4(0.56 \%$ & $4 / 4(100 \%)$ & 0.56 \\
Grupo 9 & $33(4.6 \%)$ & $33 / 33(100 \%)$ & 4.6 \\
Grupo 10 & $20(3.54 \%)$ & $20 / 25(80 \%)$ & 2.83 \\
Total & 706 & $461 / 706$ & 65.29 \\
\hline
\end{tabular}

El $47 \%$ fueron primíparas y el 53\% multíparas. En el $62.2 \%$ de los casos el trabajo de parto se inició espontáneamente, en el $19.9 \%$ se realizó inducción del trabajo de parto, y de estos, el 17.9\% correspondió a malas condiciones para inducto-conducción. El $91.78 \%$ de los fetos se encontraban en presentación cefálica al momento de parto. El $42.49 \%$ de las pacientes presentaron antecedente de cicatriz uterina.

La distribución por grupos clínicos de Robson y sus respectivas tasas de cesáreas se resumen en la tabla 1.

La indicación para interrumpir el embarazo por vía abdominal representó el $67 \%$ por causa materna y el $33 \%$ por causa fetal. La causa materna más frecuente fue cesárea iterativa, con el $42 \%$, seguida de desproporción cefalopélvica con el 38\% (Fig. 1). La causa fetal más frecuente fue distocia de presentación en un $62 \%$ (Fig. 2).

\section{Discusión}

En los últimos años se ha observado un incremento en la proporción de cesáreas en la mayoría de los países, practicándose aproximadamente 18.5 millones de cesáreas anuales, de las cuales la mitad se consideran innecesarias. En México se reportó en 1990 una tasa del 19\%, que se incrementó al 45\% en el año 2009.

La aplicación del modelo de clasificación de Robson permitió identificar cuáles son los grupos que más están aportando a la proporción de cesáreas en

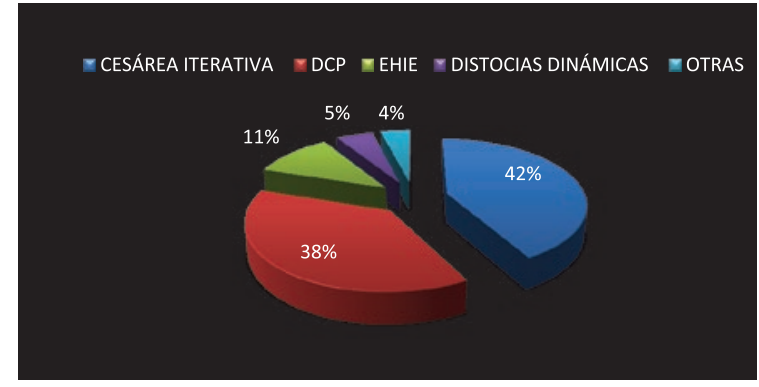

Figura 1. Causas maternas diagnosticadas para interrupción del embarazo por cesárea (\%). DCP: desproporción cefalopélvica; EHIE: enfermedad hipertensiva inducida por el embarazo.

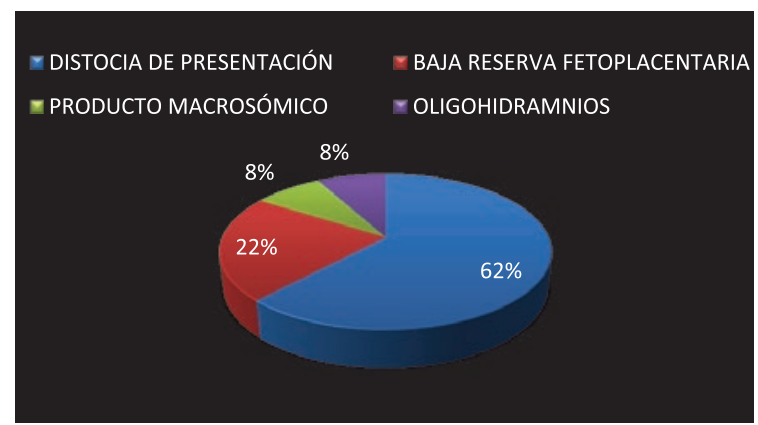

Figura 2. Diagnósticos fetales que indicaron la interrupción del embarazo por vía abdominal (\%).

el hospital, superando el porcentaje del 10-15\% establecido por la OMS. En nuestro hospital se determinó una incidencia del $65.29 \%$ por arriba de lo normado por la colectividad internacional, ocasionando un costo excesivo a lo esperado para la atención de un nacimiento. Así mismo, se ha demostrado que una tasa de cesárea superior al $10 \%$ no está asociada con 
una reducción en las tasas de mortalidad materna y neonatal. Las cesáreas pueden provocar complicaciones y discapacidades significativas, a veces permanentes, e incluso la muerte, especialmente en los lugares donde se carece de instalaciones o de la capacidad del personal de salud para realizar cirugías de forma segura y para tratar las complicaciones quirúrgicas. Idealmente, las cesáreas deben realizarse solo cuando son necesarias por razones médicas.

Los grupos que aportaron mayor número de cesáreas en la institución fueron el 5 (21.24\%), el 2 (13.88\%) y el $1(9.6 \%)$. Las principales indicaciones de cesárea encontradas fueron cesárea iterativa (42\%) y desproporción cefálico-pélvica (38\%). El grupo 5 , que es el que más aporta a la tasa global de cesárea de esta institución, podría ser modificado si se estableciera un protocolo de atención de parto vaginal después de una cesárea. En cambio, las mujeres con feto en podálica (grupos 6 y 7) y embarazo gemelar (grupo 8) generalmente tienen una mayor probabilidad de parto por cesárea, junto con las que presentan fetos en situación transversa (grupo 9), que tienen indicación obstétrica clara para el nacimiento por cesárea. La cantidad de mujeres pertenecientes a estos grupos es minoritaria dentro de nuestra institución, y el aporte a la cifra global de cesáreas es inferior al de otros grupos con menos riesgo inherente de requerir parto operatorio.

Basándonos en los hallazgos encontrados se propone al interior de la institución dar a conocer los presentes resultados, y para estudios futuros revisar las indicaciones y protocolos de inducción y conducción del trabajo de parto, uso del partograma y monitorización del bienestar fetal, las indicaciones para realizar cesárea electiva anteparto y la definición de inducción fallida. En este mismo sentido, se sugiere la adopción y la implementación de protocolos de maduración cervical, atención de parto vaginal después de cesárea y, con un menor impacto esperado, protocolos de atención de parto en presentación de nalgas. Finalmente, es posible implementar estrategias para la reducción de la tasa de cesáreas, como una segunda opinión de expertos antes de realizar el procedimiento. La información correcta concerniente a la edad gestacional y la indicación de la inducción del trabajo de parto, la disminución del número de cesáreas previas en las mujeres con este antecedente, la descripción de la indicación obstétrica o perinatal de la cesárea en los partos pretérmino, y el reporte uniforme del bienestar fetal por ultrasonido y registro tococardiográfico, permitirán adecuar las estrategias e intervenciones para racionalizar el uso de la cesárea como vía de nacimiento.

\section{Conclusiones}

La clasificación de Robson permite a cualquier institución de salud identificar los grupos de mujeres que más contribuyen a la tasa de cesáreas, orienta a establecer los grupos de mujeres con características obstétricas comunes sometidas a cesárea que requieren intervención en un protocolo institucional, y da la pauta para definir conductas que impacten en la tasa global de cesáreas en la institución.

Los grupos de mujeres con tasas específicas más altas de cesárea son aquellas con cesárea anterior (o cicatriz uterina previa), las mujeres nulíparas, especialmente las sometidas a inducción del parto y las mujeres con embarazos pretérmino. Estos grupos son susceptibles de intervención para reducir la tasa de cesáreas en cada uno de ellos e impactar la tasa global de cesáreas de la institución.

Se recomienda dar a conocer los resultados a los gineco-obstetras de la institución e implementar estrategias que permitan una reducción de la tasa global de cesáreas, aumentado la seguridad de la atención obstétrica y perinatal.

La OMS propone utilizar el sistema de clasificación de Robson como estándar global para evaluar y comparar las tasas de cesáreas, y hacer un seguimiento al respecto en los establecimientos sanitarios a lo largo del tiempo y entre ellos. Para facilitar la adopción de la clasificación de Robson por parte de los centros sanitarios, la OMS desarrollará guías de uso, implementación e interpretación, que incluirán la estandarización de términos y definiciones.

\section{Bibliografía}

1. Palean L, Gibbons L, Chacón S, Ramil V, Belizan J. Tasa de cesáreas en dos hospitales privados con normativas diferentes: abierto y cerrado. Ginecol Obstet Mex. 2012;80:263-9.

2. Ruiz SJ, Espino SS, Vallejos PA, Duran AL. Cesárea: tendencias y resultados. Perinatol Reprod Hum. 2014;28:33-40.

3. Vélez PE, Tovar GVJ, Méndez VF, López LCR, Bustos ER. Incidencia, indicaciones y complicaciones de la operación cesárea en el Hospital de Ginecopediatría del IMSS de Hermosillo, Sonora. Bol Clin Hosp Infant Edo Son. 2012;29:58-64.

4. Schnapp CS, Sepúlveda E, Robert SJA. Operación cesárea. Rev Med Clin Condes. 2014;25:987-92.

5. Cabeza VPJ, Calvo PA, Betrán AP, Mas MMM, Febles BMM, Alcocer PX, et al. Clasificación de cesáreas por grupos de Robson en dos periodos comparativos en el Hospital de Manacor. Prog Obstet Ginecol. 2010;53:385-90

6. Kazmi T, Saiseema S, Khan S. Analysis of cesarean section rate-according to Robson's 10-group classification. Oman Med J. 2012;27: 425-7.

7. Barcaite E, Kemekliene G, Railatete R, Bartuservicius A, Maleckliene L, Naisanskiene R. Cesarean section rates in Lithuania using Robson ten group classification system. Medicine. 2015;51:280-5. 
8. Farine D, Shepherd D, Robson M. Classification of cesarean sections in Canada: the modified Robson criteria. J Obstet Gynaecol Can. 2012; $34: 1133$

9. Ferreira EC, Costa ML, Cecattli JG, Haddad SM, Parpinelli MA Robson MS. Robson ten group classification system applied to woman with severe maternal morbidity. Birth. 2015;42:1

10. Betrán AP, Vindevoghel N, Souza JP, Guimezoglua AM, Torioni AM, Bhattacharya S. A systematic review of the Robson classification for cesarean section: what works, doesn't work and how to improve it. PLoS One. 2014;9:e97769.

11. Litorp H, Kidanto HL, Nystrom L, Darje E, Essen B. Increasing caesarean section rates among low-risk groups: a panel study classifying deliveries according to Robson at a University hospital in Tanzania. BMC Pregnancy. 2013;13:1-10.

12. Vogel JP, Betrán AP, Vindevoghel N, Souza JP, Torloni MR, Zhang J, et al. Use of the Robson classification to assess caesarean section trends in 21 countries: a secondary analysis of two WHO multicounty surveys. Lancet Global Health. 2015;3:260-7.

13. Betrán AP, Torloni MR, Zhang JJ, Guimezoglu AM, for the WHO working group on caesarean section. WHO statement on caesarean section rates. BJOG. 2016;123:667-70.
14. Muñoz-Enciso JM, Rosales Anjang E, Domínguez-Ponce G, Serrano-Díaz CL. Operación cesárea: ¿indicación justificante o preocupación justificada? Ginecol Obstet Mex. 2011;79:67-70.

15. Cyr RM. Myth of the ideal cesarean section rate: commentary and historic perspective. Am J Obstet Gynecol. 2006;194:932-6.

16. Zhang J, Troendle J, Reddy UM, Laughon SK, Branch DW, Burkman R, et al. Contemporary cesarean delivery practice in United States. Am J Obstet Gynecol. 2010; 203:326.e1-10.

17. Betrán AP, Vindenvoghel Nadia, Souza JP Gulmezoglu M, Torloni MR, et al. A systematic review of the Robson classifications for cesarean section. PLoS One. 2014;9:e97769.

18. Van Dillen J, Diesch M, Schutte J, Zwart J, Wolterbeek R, van Roosmalen J. Comparing grades of urgency for classification of cesarean delivery. Int J Gynaecol Obstet. 2009;107:16-8.

19. Althabe F, Belizán JM, Villar J, Alexander S, Bergel E, Ramos S, et al. Mandatory second opinion to reduce rates of unnecessary caesarean sections in Latin America: a cluster randomised controlled trial. Lancet. 2004;363:1934-40

20. Anderson GM, Lomas J. Determinants of the increasing cesarean birth rate. Ontario data 1979 to 1982 . N Engl J Med. 1984;311:887-92. 